\title{
Message from the Editors to our US and International Reviewers
}

John H. Noseworthy, $\mathrm{MD}$

Editor-in-Chief

Robert A. Gross, MD, $\mathrm{PhD}$

Deputy Editor

Andrew G. Engel, MD

Karen C. Johnston, MD, MSc

David S. Knopman, MD

Jonathan W. Mink, $\mathrm{MD}, \mathrm{PhD}$

Richard M. Ransohoff, MD

Ryan J. Uitti, MD Associate Editors
Neurology ${ }^{\circledR}$ received 1,785 new and 497 revised manuscripts (including about 42 new and 11 revised fulllength articles per week) from October 1, 2007, through March 31, 2008. During this period, a total of 3,254 reviews were received. Reviews continue to arrive within 8 days; the average time from submission to first decision for papers chosen for review was 31 days compared to 37 days in this same period during 2006-7. Because we are limited in the number of pages we can publish, we are able to accept only about $13 \%$ of full-length papers and $12 \%$ of Clinical/Scientific Notes for publication. Thank you for making specific comments regarding uniqueness of study populations, novel methods, studies that are especially educational, or new strategies for diagnosing and treating neurologic disease. These specific comments in your reviews greatly assist us in making our decisions.

Neurology and the Editors are grateful for your dedication to the Journal, which helps us fulfill our goal of publishing the articles that will most benefit authors and readers of Neurology and improve patient care. In appreciation of your efforts, we grant an hour of CME credit, if requested, for each manuscript you review for the Journal (maximum of 15 credits per year as determined by ACCME). The following criteria will determine whether credit will be granted: "it should be evident that the reviewer has read and understood the content of the manuscript, accompanying figures and tables, supplementary material to be published on the Web site, and references. To receive credit, the reviewer must make comments to the Editor that aid him/her in making an informed decision regarding publication of the manuscript and make substantive suggestions to the author." Credit is not granted for re-reviews unless the review takes more than one hour and substantial comments are made for the authors.

The reviewers listed below with one asterisk have reviewed 5 or more papers. Two asterisks indicate that the reviewer has reviewed 10 or more manuscripts. This list includes those reviewers who returned a review or reviews of initial submissions (re-reviews of the same manuscript are not included) between October 1, 2007, and March 31, 2008.

Johan A. Aarli
Dag Aarsland *
Nada Abou Fayssal
Bassel W. Abou-Khalil
Lauren E. Abrey*
Gyula Acsadi
Harold P. Adams*
Charles H. Adler *
J. Eric Ahlskog*
Allen J. Aksamit
Alberto Albanese
James W. Albers
Steven M. Albert
Megan Christine Alcauskas
Ammar Al-Chalabi
Michael P. Alexander
Andrei V. Alexandrov *
Richard P. Allen
Cargill H. Alleyne
Eric Altschuler
Anthony A. Amato **
Melissa Amick
Kimberly Amrami

George Bartzokis
Joshua I. Barzilay
Rifaat M. Bashir
Jeffrey J. Bazarian
Carl W. Bazil
Christian Beaulieu
Christopher Beck*
James T. Becker
Werner J. Becker
Raed Behbehani
Brian Bell
Michael Benatar
Oscar R. Benavente
Selim R. Benbadis
Martin Bendszus
Ralph H. Benedict
Curtis Benesch
Gary Bennett
Jeffrey L. Bennett
Susan E. Bennett
Richard Beresford
Anne T. Berg
Daniela Berg


Michel J. Berg*

Donna C. Bergen

Joseph R. Berger*

Thomas Berger*

Gregory K. Bergey

Jonas Bergquist

Samuel F. Berkovic

Robert A Bermel

James L. Bernat*

Kent C. Berridge

Elizabeth Berry-Kravis

Enrico Bertini

Frank Besag

Christopher T. Bever, Jr.

David Q. Beversdorf

Kailash P. Bhatia

Bibiana Bielekova

Christian G. Bien

Marcelo Eduardo Bigal

Kevin Biglan

Erin D. Bigler

José Biller

Valerie Biousse

Gretchen Lano Birbeck

Gary Birnbaum

Alberto Bizzi

Kevin J. Black

Thomas P. Bleck

Olaf Bodamer

John B. Bodensteiner

Bernadette Boder-Albala

Bradley F. Boeve

Bruno Bonetti

Vincenzo Bonifati

Carsten G. Bonnemann

David C. Bonovich

Natan M. Bornstein

E. Peter Bosch

Thomas M. Bosley

Patrick Bosque

Blaise F.D. Bourgeois

James H. Bower

John V. Bowler*

Geraldine B. Boylan

Kevin B. Boylan

Allison Brashear

John C. S. Breitner

Monique M. B. Breteler

Alexis Brice

Tom Britton

Bruno Brochet

Henry Brodaty

Joseph P. Broderick

Mark B. Bromberg

Edward Bromfield

David J. Brooks

Murray G. Brown

Paul W. Brown

Robert Brown

Askiel Bruno
Ellen Bubrick

Jeffrey R. Buchhalter

Aron S. Buchman

Andrew E. Budson

Dennis R. Buis

F S. Buonanno

Arthur Burghes

David J. Burn

Jeffrey M. Burns

Rami Burstein

Ashley I. Bush

Katharine Bushby

Cheryl D. Bushnell

Neil A. Busis

Rajani Ruth Caesar

J. Gregory Cairncross

Nigel J. Cairns

Paolo Calabresi

Franca Cambi

Richard M. Camicioli

Stephen C. Cannon

Roberto Cantello

Louis R. Caplan

Barbara Caracciolo

Michelle C. Carlson

Cynthia M. Carlsson

Jonathan Carr

James E. Carroll

Jonathan L. Carter

Robert A. Casero

Fernando Cendes

Hugues Chabriat

David A. Chad

David W. Chadwick

Colin Chalk *

Marc C. Chamberlain *

Piu B. Chan

Richard K. Chan

Phillip F. Chance

Linda Chang

Seemant Chaturvedi *

Jamel Chelly

Donna Chen

Honglei Chen

Robert Chen

Tony Hsiu-Hsi Chen

Neil Cherian

William P. Cheshire

Raymond T.F. Cheung *

Luisa Chiapparini

Marc I. Chimowitz

Patrick F. Chinnery

Adriano Chio

Tanuja Chitnis

Andrew J. Church

David X. Cifu

Paola Cinque

Joseph Classen

Paula R. Clemens

David B. Clifford
Christopher S. Coffey

Bernard Cohen

Jeffrey A. Cohen

Jeffrey Allen Cohen

Ronald A. Cohen

Andrew J. Cole

John W. Cole

Cynthia L. Comella

Christopher Commichau

Mark Cookson *

Giovanni Coppola

Alastair J. Corbett

James J. Corbett

John R. Corboy

Elizabeth Hedlund Corder*

Jody Corey-Bloom

Wayne T. Cornblath

Deborah A. Cory-Slechta

H. Branch Coslett

James R. Couch, Jr.

Bruce M. Coull *

Timothy J. Counihan *

Suzanne Craft

Arthur Craig

Thomas O. Crawford

Marco Crimi

Peter B. Crino

Anne H. Cross

Merit E. Cudkowicz

Gary R. Cutter

Kirk R. Daffner

Carl G.H. Dahlof

Josep O. Dalmau

Basil T. Darras

Jean-Francois Dartigues

Richard M. Dasheiff

Jasper R. Daube

Martin Daumer

Nick Davies

Karen D. Davis

Larry E. Davis

Ted M. Dawson

John W. Day

Harald De Cauwer

Philip Lawrence De Jager

Jacques De Keyser

Frank-Erik De Leeuw

Nicola De Stefano *

Marianne de Visser

Rashid Deane

Charles S. DeCarli

Christopher M. DeGiorgio

Steven T. DeKosky

Gregory del Zoppo

Norman R. Delanty

Martin B. Delatycki *

Henri J. Delecluse

Bart M. Demaerschalk

Anita DeStefano

Orrin Devinsky
Richard B. Dewey, Jr.

Stephen Dewhurst

Feza Deymeer

Salvatore Di Mauro

Alessandro Di Rocco

Ramon Diaz-Arrastia

Martin Dichgans

Bradford Clark Dickerson

Dennis W. Dickson

Hans-Christoph Diener

Marianne Dieterich

Francis J. Dimario

Michael N. Diringer*

William B. Dobyns

David W. Dodick

Okan Dogu

Dana O. Doheny

P. Murali Doraiswamy

E. Ray Dorsey

Richard Doty

Daniel B. Drachman

Jens P. Dreier

Robert Drolet

Peter D. Drummond

Ranjan Duara

Richard M. Dubinsky

Michael S. Duchowny

John Duda

Charles J. Duffy

Carole Dufouil

Aaron Dumont

Peter James Dyck **

P. James B. Dyck

Mark Eric Dyken

George C. Ebers

Scott Eggers

Florian S. Eichler

David Eidelberg

Rodger J. Elble

Jeffrey M. Ellenbogen

Lawrence W. Elmer

Murat Emre

Jerome Engel, Jr.

Sebastiaan Engelborghs

Andrew Escayg

Gail Eskes

Patricio S. Espinosa

Jonathan Evans

Amelia Evoli *

Toufic Fakhoury

John Y. Fang

Martin R. Farlow

Matthew Farrer

Hassan M. Fathallah-

$$
\text { Shaykh }
$$

Raymond Edward Faught, Jr.

Pierre Fayad

Franz Fazekas

Andrew Feigin

Valery L. Feigin

Kevin J. Felice

Lesley K. Fellows

Russ J. Ferland

Tanis J. Ferman

Hubert H. Fernandez

Jose M. Ferro

A. James Fessler

David M. Ficker

Terry D. Fife

Denise Figlewicz

Massimo Filippi *

Gerda Fillenbaum

Christopher M. Filley

John K. Fink

John N. Fink*

Alan G. Finkel

Michael F. Finkel

Glen R. Finney

Mirta Fiorio

Elizabeth Fisher

Robert S. Fisher

John D. Fisk

Kevin Flanigan

Adam S. Fleisher

Mary Kay Floeter

Tatiana Foroud

Peter A. Forsyth

Norman L. Foster

Nathan B. Fountain

Samuel A. Frank

Gary M. Franklin

David Neal Franz

Laura Fratiglioni

Mark S. Freedman

Roy Freeman

Jacqueline French

Joseph H. Friedman *

Giovanni B. Frisoni

Elliot Mark Frohman

Matthew P. Frosch

Richard Eugene Frye

Denson G. Fujikawa

Hidenao Fukuyama *

Cindy J. Fuller

Karen L. Furie

Yoshiaki Furukawa

Eija Gaily

Peter Gal 
Barry E. Gidal

Donald L. Gilbert

Donald H. Gilden *

Sudeep S. Gill

Frank Gilliam

Ian Gilron

Arthur Ginsberg *

Gavin Giovannoni

Jonathan D. Glass *

Thomas H. Glick

M. Maria Glymour

Norbert Goebels

Christopher G. Goetz

Lawrence I. Golbe

Ralf Gold

Myla Goldman

Meredith Golomb

Paul T. Golumbek

Christopher M. Gomez

Ramon Gilberto Gonzalez

Pedro Gonzalez-Alegre

Howard Parker Goodkin *

J. Clay Goodman

Paul H. Gordon

Maria Luisa Gorno-

Tempini

Kenneth C. Gorson *

Jean Gotman

William D. Graf*

Neill R. Graff-Radford

Francesc Graus

Chris S. Gray

J. Timothy Greenamyre

Steven A. Greenberg

Paul Greene

Robert S. Greenwood

David Matthew Greer

Jennifer J. Griggs

Robert C. Griggs

Gary Gronseth

Toby I. Gropen

James C. Grotta

Michael Gruenthal *

Renzo Guerrini

Christian Guilleminault

Rishi Gupta

David H. Gutmann

Ludwig Gutmann *

Katrina A. Gwinn

Mary N. Haan *

Angelika F. Hahn

Cecil David Hahn

E. Clarke Haley, Jr. **

Michael Gabor Halmagyi

John J. Halperin

Adrian Handforth

Michael Hanna

Orla Hardiman

John Hardy

Judith U. Harrer
Robert G. Hart

Adam L. Hartman

Hans-Peter Hartung

Norman James Haughey

Robert A. Hauser

W. Allen Hauser

Sheryl R. Haut

Peter Hedera *

Kenneth M. Heilman

J. Claude Hemphill

John W. Henson

Susan T. Herman

Ulrich Herrlinger

David N. Herrmann

Andrew D. Hershey

Linda A. Hershey

Dale Hesdorffer

David C. Hess

Daniel B. Hier

Joseph James Higgins

Argye Elizabeth Hillis

David Hilton-Jones

Judith A. Hinchey

Rogier Q. Hintzen

Michio Hirano

Shu Leong Ho

Timothy F. Hoban

Jeremy C. Hobart

Eric P. Hoffman

David B. Hogan

Katherine Holland

Neil R. Holland

Robert G. Holloway

Fay B. Horak

Martin W.I.M. Horstink

Gang $\mathrm{Hu}$

Katharina Hüfner

R.A.C. Hughes

Jacques Hugon

Jill Hunter

Christopher Hyson

Susan T. Iannaccone

Shu-ichi Ikeda

KyoungBin Im

Paul G. Ince

Matilde Inglese

Fabio M. Iwamoto *

Michael Jacewicz

Caroline Jackson

Christian Jacobi

Bradley S. Jacobs

Teresa L. Jacobs

Steven Jacobson

Allan S. Jaffe

William J. Jagust

Lara E. Jeha

Joanna C. Jen

Mary Jensen

Shafali Jeste *

Nathalie Jette
Gregory A. Jicha

Hershel Jick

Yaping Jin

Hyder Azad Jinnah

Julene K. Johnson

Keith A. Johnson

Steven W. Johnson

Lori C. Jordan

Ralph F. Jozefowicz

Burk Jubelt

Vern C. Juel

Csaba Juhasz

Hannu Kalimo

David F. Kallmes

Lalit Kalra

Henry J. Kaminski *

Kousuke Kanemoto *

Andres M. Kanner

Petr Kanovsky

Kejal Kantarci *

Orhun H. Kantarci

Ludwig Kappos

Jaideep Kapur

George Karpati

Carlos S. Kase

Scott E. Kasner

Bashar Katirji

Douglas Katz

Daniel I. Kaufer

David I. Kaufman

Mark Kaufman

Anthony Kaufmann

Harish C. Kavirajan

Hideshi Kawakami

Claudia Kawas

Edward M. Kaye

Brendan J. Kelley

David Kent

Thomas A. Kent

Kevin A. Kerber

Walter N. Kernan

Douglas A. Kerr

Jurg Kesselring

Jaffar Khan

Omar A. Khan *

Karl D. Kieburtz

Bernd C. Kieseier

Ronald J. Killiany

Jong S. Kim

Scott Kim

Jun-ichi Kira

Heidi Kirsch

Howard S. Kirshner

John T. Kissel *

Brett Kissela

Steven J. Kittner

Nefati Kiylioglu

Caroline Klein

Christopher J. Klein

Pavel Klein
Dawn Kleindorfer

Thomas Klopstock

William Knight

Robert Knowlton

Alana Knudson

Eliane Kobayashi

Marcus W. Koch

Peter. J. Koehler

Dennis Kolson

Barbara S. Koppel

Igor J. Koralnik

Amos D. Korczyn

H. Kowa

Joel Kramer

Gregory L. Krauss

Joachim K. Krauss

Lauren B. Krupp

Christian Kubisch

Walter A. Kukull

Lewis H. Kuller

Dimitri Kullmann

Neeraj Kumar

Rajeev Kumar

Mark J. Kupersmith

Tobias Kurth *

John F. Kurtzke

Abraham Kuruvilla

Patrick Kwan

Jennifer M. Kwon

Daniel L. Labovitz

Pascal Laforêt

Andre H. Lagrange

Rose K. Lai

Bethan Lang

John T. Langfitt

Peter Langhorne

Maarten G. Lansberg

Douglas J. Lanska

Dan Larriviere

Andrew B. Lassman

Norman Latov

Leonore J. Launer

Robert B. Layzer

Ronald M. Lazar

Holly Ledyard

Andrew J. Lees

Frank Lehmann-Horn

R. John Leigh

Enrique C. Leira

Frederick A. Lenz

Holger Lerche

Norma B. Lerner

Ronald P. Lesser

Mark Lew

Richard A. Lewis

Peter A. LeWitt

Didier Leys

Ge Li

Yi Li

David S. Liebeskind

Jane L. Liesveld

E. Paul Lindell

Lynda D. Lisabeth

Robert P. Lisak

Irene Litvan

Lynn Liu

Eric L. Logigian

Ivan S. Login

W.T. Longstreth, Jr. *

Oscar L. Lopez

David W. Loring

Jau-Shin Lou

Elan D. Louis

Jose Luchsinger

Albert C. Ludolph

Helmi L. Lutsep

Lienhard Maeck

Mark W. Mahowald

Caterina Mainero

Eugene O. Major

Michael Makdissi

Pauline M. Maki

Paul F. Malloy

Edward Manno

Javier Mar

Demetrius M. Maraganore

Carole L. Marcus

Ron Margolis

William R. Markesbery

William Marks

Connie Marras

Maria Giovanna Marrosu

Frederick J. Marshall

Randolph S. Marshall

Wayne Martin *

Rudolf Martini

Ralph N. Martins

Joseph C. Masdeu

Janice M. Massey

David B. Matchar

Farrah J. Mateen

Jean K. Matheson

David H. Mattson

Mark P. Mattson

Arne May

Stephan A. Mayer

Justin C. McArthur

Michael P. McDermott*

William M. McDonald

Martin J. McKeown * 
Andreas MeyerLindenberg Mohamad Mikati Jamal A. Mikdashi John W. Miller Joshua W. Miller Robert G. Miller

Scott Mintzer

Susumu Miyamoto

Yoshikuni Mizuno

J. P. Mohr

Xavier Montalban

Thomas J. Montine

Lauren R. Moo

Ann Moormann

Paul Moots

Lewis B. Morgenstern *

Jean Morrisey

Leslie Morrison

James A. Mortimer

Solomon L. Moshe

Thomas H. Mosley

Mark L. Moster

Dwight E. Moulin

M. Maral Mouradian

Ulrich Müller *

Daniel L. Murman

Anne M. Murray

Gary J. Myers

Richard H. Myers

Stephen E. Nadeau

Nobukazu Nakasato

Avindra Nath

Bart Nathan

Edwin M. Nemoto

Jayne Ness

Peter J. Nestor

Jeff L. Neul

Nancy J. Newman

David E. Newman-Toker

Garth A. Nicholson

MingMing Ning

Ichizo Nishino

Michael J. Noetzel

Yoshiko Nomura

Gustavo A. Nores

Kathryn N. North

Katia Noyes

Marc Nuwer

Anne Louise Oaklander

Thomas O. Obisesan

Paul O'Connor

Wolfgang H. Oertel

Joel J. Oger

Jeffrey G. Ojemann

Anders Oldfors

John M. Olichney

William G. Ondo

Brian P. O'Neill

Stephen M. Oppenheimer
Richard Orrell

Padraig O'Suilleabhain

Brian R. Ott

Bruce Ovbiagele

Ryan Taylor Overman *

Laurie J. Ozelius

Andrew R. Pachner

Alison M. Pack

Luca Padua

Rajesh Pahwa

Aarno Palotie

Massimo Pandolfo

Hillel Panitch

Leonardo Pantoni

Gabriel Pardo

Davide Pareyson

Alvaro Pascual-Leone

Giulio Maria Pasinetti

Maria A. Pastor

Pau Pastor

Damon Patterson

Marc C. Patterson

Shanna K. Patterson

Steven G. Pavlakis

Valory N. Pavlik

Haydeh Payami

Page B. Pennell

Patricia E. Penovich

Alan K. Percy

Edward Perl

Susan L. Perlman

George Perry

B. Lee Peterlin

Charles Peters

Ronald C. Petersen

George W. Petty

Alessandro Pezzini

Michel Philippart

Lawrence H. Phillips, II

Daniela Pilz

Istvan Pirko

Sean J. Pittock

Gordon T. Plant

Brenda L. Plassman

David Pleasure

Samuel Pleasure

Chris H. Polman

David Polston

Michael Polydefkis

Scott L. Pomeroy

John D. Port

William J. Powers

Sashank Prasad *

Daniel Z. Press

Niels Prins

Michael Privitera

J. Javier Provencio

Amy A. Pruitt

Louis J. Ptacek

Patrick M. Pullicino
R. Allan Purdy

Timothy Quill

Joseph Quinn

Adnan I. Qureshi

Alejandro A. Rabinstein *

Michael K. Racke

Michael S. Rafii

Ann B. Ragin

Alexander H. Rajput

Michele Lyn Rajput

V. Ramachandran

Nabih M. Ramadan

Isabelle Rapin

Alan M. Rapoport

Bernard Ravina

G.V. Raymond

Lawrence D. Recht

Bruce R. Reed

Stephen G. Reich

Harold L. Rekate

Norman R. Relkin

Susan M. Resnick

Nancy D. Richert

Jack E. Riggs

Trond Riise

David Riley

John M. Ringman

James J. Riviello

Matthew Rizzo

William B. Rizzo

E. Steve Roach

Kenneth Rockwood

Robert L. Rodnitzky

Ekaterina A. Rogaeva

Gustavo C. Roman

Jose Rafael Romero

Jonathan Rosand

H. Diana Rosas

Michael R. Rose

Gary A. Rosenberg

Adam Rosenblatt

Myrna R. Rosenfeld

David B. Rosenfield

Elizabeth Ross

Owen A. Ross

Michael L. Rossen

Andrea O. Rossetti

Natalia S. Rost

Todd D. Rozen

Udo Rüb

Richard A. Rudick

Robert L. Ruff

Tatjana Rundek

Barry S. Russman

Paul Rutecki

Ann Saada

Marwan N. Sabbagh

Ralph L. Sacco

Dessa Sadovnick

Zarife Sahenk
Gerard Said

Ken Sakaie

Stephen P. Salloway

Martin A. Samuels

Dirk Sander

Paola Sandroni

Mary Sano

Filippo M. Santorelli

Susumu Sato

Rachel J. SaundersPullman

Jeffrey L. Saver

Nikolaos Scarmeas

Elio Scarpini

Ingrid E. Scheffer

Huub Schellekens

Ann I. Scher

Wouter Ingmar Schievink

David Schiff*

Raphael Schiffmann

Bradley L. Schlaggar

Jeremy D. Schmahmann

Frederick A. Schmitt

Heike I. Schmolck

Julie A. Schneider

Susanne A. Schneider

Ronald Schondorf

Norbert Schuff

Gerd Schulte-Körne

Maria T. Schultheis

Markus Schürks

Jason M. Schwalb

Lee H. Schwamm

Steven R. Schwid *

Thomas F. Scott

Meghan M. Searl

William W. Seeley

Alan Z. Segal

Takashi Seki

Yojiro Seki

Duygu Selcen

Finn Sellebjerg

Warren R. Selman

Ola A. Selnes

Linda M. Selwa

Alessandro Serra

Sudha Seshadri

Saad Shafqat

Kathleen M. Shannon

James A. Sharpe

Tarek Sharshar

Volney L. Sheen

Jeremy M. Shefner

Elliott Sherr

Raj D. Sheth

Michael I. Shevell

Dean Shibata

Jerry J. Shih

Joshua S. Shimony

Robert K. Shin

Shlomo Shinnar

Eric Shoubridge

Ira Shoulson

David Shprecher

Lisa M. Shulman

Michael E. Shy

Andrew D. Siderowf

John J. Sidtis

Bruce Sigsbee

Michael H. Silber

Howard J. Silberstein

Stephen D. Silberstein *

Isabel Silveira

Zachary Simmons

David M. Simpson

Carlos Singer

Aneesh B. Singhal

Andrew B. Singleton

Michael Sinnreich

Joseph I. Sirven

John T. Sladky

A. Gordon Smith

Benn E. Smith *

Glenn E. Smith

Stephen A. Smith

Kathleen A. Smyth

Barry J. Snow

Elson L. So

Norman K. So

Hilkka Soininen

Giancarlo Solaini

Nina Solenski

Norbert Sommer

Per Sorensen

Eric J. Sorenson

Roderick Spears

Salvatore Spina

Anne Spurkland

Rosanna Squitti

Carl E. Stafstrom

David G. Standaert

Sidney Starkman

Robert Staudinger

Israel Steiner

Barney J. Stern

Yaakov Stern

Annette Sterr

Glen H. J. Stevens

Suzanne Stevens

Jonathan Stewart 
Gene Sung

William Sutherling

Jerry W. Swanson *

Michael Swash **

Kathryn J. Swoboda

Minoru Tagawa

Michele Tagliati

Eng-King Tan

Naoyuki Taniguchi

Mark Tarnopolsky

Turgut Tatlisumak

Carlos Bernardo Tauil

Rabi Tawil

Charles H. Tegeler

Richard B. Tenser

Kenshi Terajima

G. M. Terwindt

William H. Theodore

Vincent N. Thijs

Liu Lin Thio

Maria Thom

Alan J. Thompson

Charles A. Thornton

Pentti J. Tienari

Mary C. Tierney

Gretchen E. Tietjen

Vincent Timmerman

Maja Tippmann-Peikert

Eduardo F. Tizzano
Eduardo S. Tolosa

Torbjörn Tomson

Haluk Topaloglu

Michel T. Torbey

James C. Torner

Klaus V. Toyka

Bryan J. Traynor

Helen Tremlett

S. Anand Trip

Alexander I. Tröster

Alex Tselis

Georgios Tsivgoulis *

Debby W. Tsuang

Stanley Tuhrim

Paul Tuite

William R. Tyor

Ken Uchino

Bjarne Udd

Victor C. Urrutia

Nachum Vaisman

Massimiliano Valeriani

Giuseppe Vallar

Josep Valls-Sole

Hans Van Bokhoven

Christine Van Broeckhoven

Marjo S. Van Der Knaap

J. Gert Van Dijk

Cornelia M. Van Duijn
Jay A. Van Gerpen

Jacobus J. Van Hilten

Gregory P. Van Stavern

John C. Van Swieten

Edward Vates

Shannon L. Venance

Joe Verghese

Steven Vernino

Meike W. Vernooij

Alberto Verrotti

Barbara G. Vickrey

Matti Viitanen

Harry V. Vinters

Francesco Violi

Pieter Jelle Visser

John Vissing

Anand Viswanathan

Jens Volkmann

Jean Paul Vonsattel

Pieter E. Vos

Rhonda R. Voskuhl

Tiffini S. Voss

Andrew J. Waclawik

Salina P. Waddy

Thaddeus S. Walczak

Ruth H. Walker

Michael Wall

Peter Walla

Uwe Walter
Ching H. Wang

Jie Jin Wang

Thomas N. Ward

Craig Watson

James Watson

John D. G. Watson

Emmanuelle Waubant

Lawrence R. Wechsler

Daniel E. Weiner

William J. Weiner

Brian G. Weinshenker

Bianca Weinstock-

Guttman

Marc G. Weisskopf

Kenneth M.A. Welch

Patrick Y. Wen

Shawn K. Westaway

Cynthia Wetmore

Lawrence J. Whalley

Charles White

Roberta F. White

Rachel Whitmer

Jennifer L. Whitwell

Heinz Wiendl

Eelco F. M. Wijdicks *

Christine Anne Wijman

Linda S. Williams

Michael A. Williams *

Hugh J. Willison
George R. Wilmot

Robert S. Wilson

Janet L. Wilterdink

Dean M. Wingerchuk

Juliane Winkelmann

John H.J. Wokke

Gil I. Wolfe

Eric T. Wong

Ka Sing Wong

Daniel Woo

Fred G. Wooten

Shirley Wray

Clinton B. Wright

George K. York

Michele York

Tarek Yousry

Nobuhiro Yuki

Nathan Zasler

Mayana Zatz

Allyson Zazulia

David S. Zee

John M. Zempel

Theresa A. Zesiewicz

Ulf Ziemann

Berislav V. Zlokovic

Douglas W. Zochodne

Richard M. Zweifler 


\title{
Neurology
}

\author{
Message from the Editors to our US and International Reviewers \\ John H. Noseworthy, Robert A. Gross, Andrew G. Engel, et al. \\ Neurology 2008;71;2-6 \\ DOI 10.1212/01.wnl.0000325018.06284.35
}

This information is current as of June 30, 2008

\section{Updated Information \&}

Services

Permissions \& Licensing

Reprints including high resolution figures, can be found at:

http://n.neurology.org/content/71/1/2.full

Information about reproducing this article in parts (figures,tables) or in its entirety can be found online at:

http://www.neurology.org/about/about_the_journal\#permissions

Information about ordering reprints can be found online:

http://n.neurology.org/subscribers/advertise

Neurology ${ }^{\circledR}$ is the official journal of the American Academy of Neurology. Published continuously since 1951, it is now a weekly with 48 issues per year. Copyright . All rights reserved. Print ISSN: 0028-3878. Online ISSN: 1526-632X.

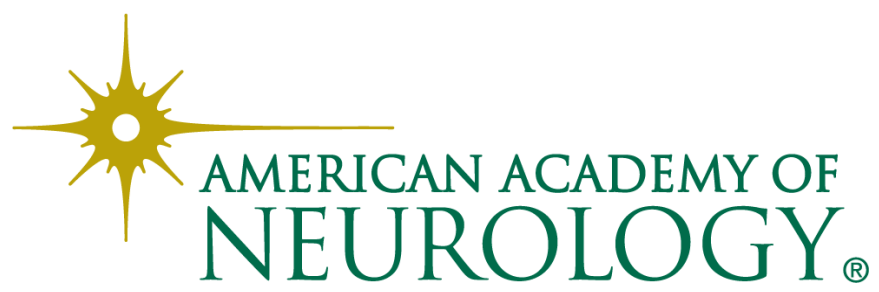

